\title{
螺旋状配位结构的碱土金属开链冠醚 三元配合物"
}

\author{
刘伟生 ${ }^{* *}$ 温永红 刘雪原 谭民裕 \\ (兰州大学化学化工学院, 兰州 730000)
}

\begin{abstract}
摘要合成并表征了 4 种碱土金属苦味酸盐与 $\mathrm{N}, \mathrm{N}, \mathrm{N}^{\prime}, \mathrm{N}^{\prime}-$ 四苯基-3,6,9-三氧杂十一烷 二酰胺(TTD) 的固态配合物 $\mathrm{M}$ (Pic) ( $_{2} \mathrm{TTD}$ (1. M = Mg, 2. M = Ca, 3. M = Sr, 4. M = Ba). 配合物 3 的结构分析表明, Sr ( II) 与 TTD 及两个二齿 Pic 配位, 配位数为 9 , 配位多面体 为变形三帽三角棱柱; TTD 作为五齿配体, 其螯合链形成右手螺旋状配位结构, 这种螯 合螺旋链具有较为固定的螺旋半径, 对金属离子有较高的配位选择性. 在结构上初步 解释了 TDD 对碱土金属离子具有高选择性的原因。
\end{abstract}

\section{关键词碱土金属 开链冠醚 配合物 螺旋状配位结构}

具有独特分子结构的酰胺型开链冠醚, 因其优异的物理、化学性质和对金属离子的选择 性配位能力而倍受人们的青睐, 这类化合物已成功地用作离子选择性电极活性物质和萃取 剂 ${ }^{[1 ~ 5]}$. 其中, $\mathrm{N}, \mathrm{N}, \mathrm{N}^{\prime}, \mathrm{N}^{\prime}$-四苯基-3,6,9-三氧杂十一烷二酰胺(TTD)对 $\mathrm{Sr}^{2+}, \mathrm{Ba}^{2+}$ 及稀土离子具有 很高的萃取率, 同时对 $\mathrm{Ba}^{2+}$ 离子具有特殊的选择性, 已用于 $\mathrm{Ba}^{2+}$ 选择电极活性物质及 $\mathrm{Ba}^{2+}$ 离子 传递剂 ${ }^{[1]}$. 近期我们探讨了开链冠醚对金属离子的选择性配位能力和构效关系, 发现此类配体 对稀土离子具有选择性配位能力的原因是形成 “类环状” 配位结构 ${ }^{[6 \sim 9]}$. 为了进一步研究此类 配体对碱土离子具有选择性配位能力的原因, 探讨构效关系, 本文报道了 4 种碱土金属苦味酸 盐与 TTD 配合物的合成与结构, 并首次在酰胺型开链冠醚配合物中发现螺旋状的配位结构, 这对于丰富配位化学的内容, 以及指导新型萃取剂的分子设计都具有重要的意义.

\section{1 实验与晶体结构分析}

\section{1 碱土金属苦味酸盐的制备}

先用碱土金属氯化物 $\mathrm{MCl}_{2}$ 与适量 $\mathrm{Na}_{2} \mathrm{CO}_{3}(\mathrm{M}=\mathrm{Ca}, \mathrm{Sr}, \mathrm{Ba})$ 溶液或 $\mathrm{NaOH}$ 溶液 $(\mathrm{M}=\mathrm{Mg})$ 反应制得 $\mathrm{MCO}_{3}$ 或 $\mathrm{Mg}(\mathrm{OH})_{2}$ 沉淀; 再将相应沉淀加人到热的苦味酸的水溶液中, 加热蒸发至溶 液中出现晶膜, 冷却, 析出晶体. 经重结晶后得到黄色晶体, 再用少量水洗涤约 10 次后, 真空 干燥 $12 \mathrm{~h}$ 得产品, 产率 $85 \%$ 以上.

\section{2 配合物及单晶的制备}

将 $0.5 \mathrm{mmol}$ 苦味酸盐溶于 $20 \mathrm{~mL}$ 无水乙醇中, 搅拌下逐滴加人含有 $0.5 \mathrm{mmol}$ TTD 的 $15 \mathrm{~mL}$ 无水乙醇溶液, 混合液中立刻有沉淀生成, 继续搅拌 4h, 将沉淀过滤并用无水乙醇洗涤 
$3 \sim 5$ 次, 于 $\mathrm{P}_{4} \mathrm{O}_{10}$ 干燥器中真空干燥 $48 \mathrm{~h}$ 得到黄色粉末状固体, 各配合物在空气中稳定. 将 $\mathrm{Ca}(\mathrm{II})$ 配合物粉末溶于乙腈, 放人未塞紧的雉形瓶中挥发近两个月后, 析出黄色的块状晶体.

\section{3 测试方法及仪器}

金属离子含量用 EDTA 配位滴定法测定, 铬黑 $\mathrm{T}$ 作指示剂, C, H, N 含量分析用德国产 Elementar Vario EL 型元素分析仪测定. 电导测定使用上海产 DDS-11A 型电导率仪. 红外光谱 用美国产 Nicolet 170SX-FT 红外光谱仪, KBr 压片，在 $200 \sim 4000 \mathrm{~cm}^{-1}$ 波段摄谱. ${ }^{1} \mathrm{H}$ NMR 谱用 Brucker AC80 核磁共振仪, 氝代丙酮作溶剂. 热分析使用国产 PCt-2 型热谱仪于静态空气中进 行, $\mathrm{Al}_{2} \mathrm{O}_{3}$ 作参比.

\section{4 配合物 3 的晶体结构测定}

选取晶粒大小为 $0.8 \mathrm{~mm} \times 0.6 \mathrm{~mm} \times 0.4 \mathrm{~mm}$ 的晶体 $\mathbf{3}$, 置于 Rigaku-AFC5R 四圆衍射仪上, 用石墨单色化的 $\operatorname{MoK} \alpha(\lambda=0.71073)$ 辐射, 以 $\omega / 2 \theta$ 扫描方式, 在 $2^{\circ} \leqslant 2 \theta \leqslant 50^{\circ}$ 范围内, 收集 到独立衍射点 9037 个, 可观察衍射点 3428 个 $[I>3 \sigma(I)]$, 所得数据经 $L P$ 因子和经验吸收校正. 晶体结构利用 TEXSAN2.0 程序解出. 由 Patterson 函数法求得 Sr 原子坐标, 经 Fourier 合成多 次迭代得到全部非氢原子坐标, 再以块对角矩阵最小二乘法纳人各向异性热参数修正至收玫. 晶体学数据: 分子式 $\mathrm{C}_{44} \mathrm{H}_{36} \mathrm{~N}_{8} \mathrm{O}_{19} \mathrm{Sr}, M r=1068.43$, 单斜晶系, $\mathrm{P} 2{ }_{1} / \mathrm{a}$ 空间群, 晶胞参数: $a=$ 1.7014(7), $b=1.5559(5), c=1.780(1) \mathrm{nm}, \beta=95.15(4)^{\circ}, V=4.694(4) \mathrm{nm}^{3}, Z=4, \mu(\operatorname{MoK} \alpha)=$ $11.95 \mathrm{~cm}^{-1}, D_{c}=1.51 \mathrm{~g} \cdot \mathrm{cm}^{-3}, F(000)=2184, R=0.059, R_{w}=0.068$.

\section{2 结果与讨论}

\section{1 配合物的组成、溶解性及热重分析}

元素分析结果(表 1)表明, 该系列配合物的组成为 $\mathrm{M}(\mathrm{Pic})_{2} \mathrm{TTD}$ ( $\mathrm{M}=\mathrm{Mg}, \mathrm{Ca}, \mathrm{Sr}, \mathrm{Ba}$ ). 所 有配合物易溶于乙腈、丙酮、甲醇, 微溶于乙醇, 难溶于苯、环己烷等. 室温下配合物丙酮溶 液 $\left(1.0 \times 10^{-3} \mathrm{~mol} \cdot \mathrm{L}^{-3}\right)$ 的摩尔电导数据表明 ${ }^{[8]}, \mathrm{Mg}($ II $)$ 的配合物 1 为 $1: 1$ 型电解质, 一个 $\mathrm{Pic}^{-}$ 处于配合物的外界; 而其余 3 个配合物均属于非电解质, 即两个 Pic 均参与配位. 这是由于 $\mathrm{Mg}^{2+}$ 半径较小, 只能形成较低配位数的配合物. 该系列配合物热分解行为相似, 在 $200^{\circ} \mathrm{C}$ 以前 均无热效应且无失重, 进一步证明配合物中不含水. 配合物无熔点, 加热至 $285^{\circ} \mathrm{C}$ 后发生剧烈 氧化分解, $\mathrm{TG}$ 曲线出现明显失重, $600^{\circ} \mathrm{C}$ 左右分解完全, 最终残余物的重量与相应氧化物的计 算值基本符合.

表 1 配合物的元素分析及摩尔电导数据

\begin{tabular}{cccccc}
\hline \multirow{2}{*}{ 配合物 } & \multicolumn{3}{c}{ 测量值(计算值) $/ \%$} & \multirow{2}{*}{ 产率 $/ \%$} & 摩尔电导 $/ \Omega^{-1} \cdot \mathrm{cm}^{-1} \cdot \mathrm{mol}^{-1}$ \\
\cline { 2 - 4 } & $\mathrm{C}$ & $\mathrm{H}$ & & $\mathrm{N}$ & 112 \\
$\mathrm{Mg}(\mathrm{Pic})_{2} \mathrm{~L}$ & $52.55(52.57)$ & $3.62(3.61)$ & $11.48(11.15)$ & & 91 \\
$\mathrm{Ca}(\mathrm{Pic})_{2} \mathrm{~L}$ & $51.68(51.76)$ & $3.51(3.55)$ & $11.03(10.98)$ & 87 & 85 \\
$\mathrm{Sr}(\mathrm{Pic})_{2} \mathrm{~L}$ & $49.33(49.46)$ & $3.35(3.40)$ & $10.55(10.49)$ & 86 & 76 \\
$\mathrm{Ba}(\mathrm{Pic})_{2} \mathrm{~L}$ & $47.21(47.26)$ & $3.28(3.25)$ & $10.05(10.02)$ & 86 & 70 \\
\hline
\end{tabular}

\subsection{IR 谱}

自由配体的有关特征红外吸收峰 $v(\mathrm{C}=\mathrm{O})\left(1686 \mathrm{~cm}^{-1}\right)$ 和 $v(\mathrm{C}-\mathrm{O}-\mathrm{C})\left(1140 \mathrm{~cm}^{-1}\right)$ 在形成配合 物后, 分别向低波数方向位移 72 74 和 57 61 $\mathrm{cm}^{-1}$, 表明羰基氧和醚氧原子均与金属离子配位. 配合物的 IR 谱中, 自由苦味酸的 $\mathrm{OH}$ 面外弯曲振动峰 $\left(1151 \mathrm{~cm}^{-1}\right)^{[9]}$ 消失, 表明碱土离子取代了 
酚差基氢原子. 苦味酸苯环上的 $v_{\mathrm{as}}\left(-\mathrm{NO}_{2}\right)\left(1555 \mathrm{~cm}^{-1}\right)$ 及 $v_{\mathrm{S}}\left(-\mathrm{NO}_{2}\right)\left(1342 \mathrm{~cm}^{-1}\right)$ 在配合物中均分 裂成双重峰, 其振动频率分别出现在 1570, 1550 $\mathrm{cm}^{-1}$ 和 1364, 1320 $\mathrm{cm}^{-1}$, 表明 $\mathrm{Pic}^{-}$中的硝 基也参与了配位 ${ }^{[9]}$. 各配合物 IR 谱中均未出现水的特征吸收峰, 说明配合物不含水, 与元素分 析结果相一致.

将该系列配合物与相应的稀土苦味酸盐配合物 ${ }^{[6,7]}$ 相比(表 2), 可以发现两系列配合物的 红外位移 $\Delta v$ 值按 $\mathrm{Y}^{3+} \rightarrow \mathrm{Er}^{3+} \rightarrow \mathrm{Eu}^{3+} \rightarrow \mathrm{La}^{3+}$ 及 $\mathrm{Mg}^{2+} \rightarrow \mathrm{Ca}^{2+} \rightarrow \mathrm{Sr}^{2+} \rightarrow \mathrm{Ba}^{2+}$ 的顺序增大, 即在所带电荷 相同的情况下, $\Delta v$ 随金属离子半径的增大而增大, $\mathrm{Ba}$ (II )配合物与 $\mathrm{La}(\mathrm{III})$ 配合物的 $\Delta v$ 最大且 几乎相等, 表明配体 TTD 与 $\mathrm{Ba}($ II $)$ 和 $\mathrm{La}($ III ) 都有很强且近似相等的配位能力, 这就解释了 TTD 对 $\mathrm{Ba}($ II $)$ 和 $\mathrm{La}$ (III) 具有配位选择性和很高萃取率 $[\mathrm{Ba}(\text { II })=99.19 \%, \mathrm{La}(\text { III })=99.05 \%]^{[4]}$ 的原因.

表 2 TTD 与碱土及稀土配合物的红外位移 $\Delta v\left(\mathrm{~cm}^{-1}\right)$

\begin{tabular}{lcccccccccc}
\hline & $\mathrm{Mg}^{2+}$ & $\mathrm{Ca}^{2+}$ & $\mathrm{Sr}^{2+}$ & $\mathrm{Ba}^{2+}$ & $\mathrm{La}^{3+}$ & $\mathrm{Eu}^{3+}$ & $\mathrm{Tb}^{3+}$ & $\mathrm{Er}^{3+}$ & $\mathrm{Y}^{3+}$ \\
\hline$v(\mathrm{c}=\mathrm{o})$ & 73 & 72 & 73 & 74 & 74 & 73 & 73 & 70 & 71 \\
$v(\mathrm{c}-\mathrm{O}-\mathrm{c})$ & 57 & 60 & 61 & 61 & 62 & 59 & 58 & 58 & 57 \\
\hline
\end{tabular}

\section{$2.3{ }^{1}$ H NMR 谱}

自由配体的 ${ }^{1} \mathrm{H}$ NMR 谱中 3 种质子的化学位移 $\delta$ 分别为 $7.38\left(\mathrm{C}_{6} \mathrm{H}_{5^{-}}, \mathrm{m}\right), 4.03\left(-\mathrm{C}(\mathrm{O}) \mathrm{CH}_{2^{-}}, \mathrm{s}\right)$ 和 $3.57\left(-\mathrm{C}_{2} \mathrm{H}_{4^{-}}, \mathrm{s}\right)$, 在配合物中均向低场移动, 其中- $\mathrm{C}(\mathrm{O}) \mathrm{CH}_{2}$-和- $\mathrm{C}_{2} \mathrm{H}_{4}$-质子的位移量 $(\delta)$ 分别为 $0.26 \sim 0.33$ 和 $0.25 \sim 0.36$, 而 $\mathrm{C}_{6} \mathrm{H}_{5}$-质子位移量 $(\delta)$ 仅为 $0.03 \sim 0.16$, 这是由于羰基氧和醚氧原子向 碱土金属离子配位后 $\mathrm{M} \leftarrow \mathrm{O}$ 键的诱导效应引起的, $\mathrm{C}_{6} \mathrm{H}_{5^{-}}$与配位原子相距较远, 受影响较小, 其 质子位移量亦较小. 自由 $\mathrm{Hpic}$ 的 $\mathrm{OH}$ 质子共振吸收峰在配合物中消失, 说明在配合物中碱土 离子已取代了酚羟基质子. 自由 Hpic 苯环上质子的化学位移 $(\delta 9.12)$ 在形成配合物后向高场移

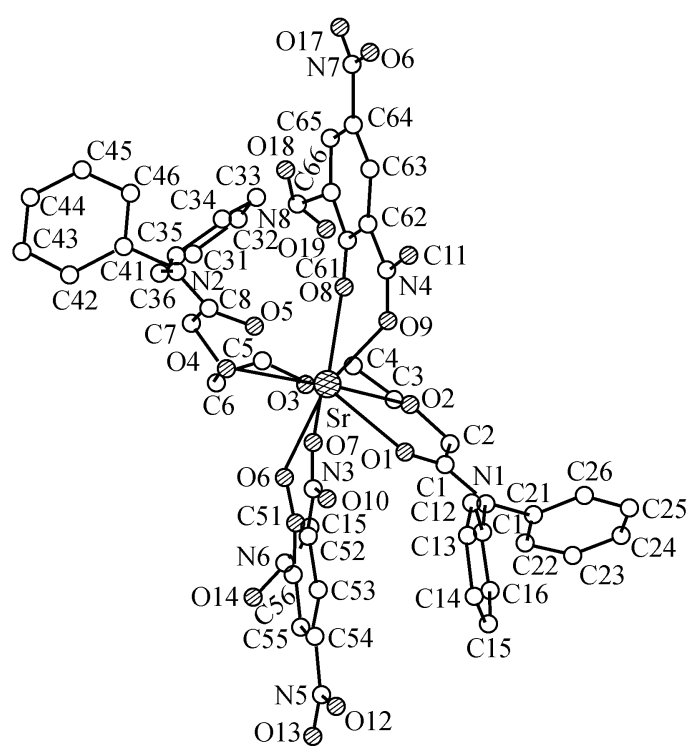

图 1 配合物 $\mathbf{3}$ 的分子结构
动 $\delta 0.40 \sim 0.45$, 这是由于当酚羟基质子被电负性 较小的碱土金属离子取代后，苯环上的电荷密 度增加，质子所受屏蔽作用增加，使其化学位移 向高场移动. 配合物 ${ }^{1} \mathrm{H}$ NMR 谱图上 $\mathrm{Pic}^{-}$苯环上 质子峰均表现为单峰, 这可能是由于在室温的 溶液中, $\mathrm{Pic}^{-}$之间作激烈化学交换的结果 ${ }^{[10]}$.

\section{4 配合物 3 的晶体结构分析}

$X$ 射线单晶测定结果表明, 配合物 $\mathbf{3}$ 组成为 [Sr(Pic) $)_{2}$ TTD], 电中性的配合物分子通过 van der Waals 力结合成晶体. 其分子结构示于图 1 , 非氢原子坐标列于表 3 , 部分键长和键角见表 4 .

TTD 的 5 个氧原子及两个双齿 $\mathrm{Pic}^{-}$的 4 个氧 原子与 $\operatorname{Sr}(I I)$ 配位, 中心离子配位数为 9 , 配位 多面体为变形三帽三角棱柱. TTD 作为五齿配体, 其螯合链形成螺旋状的配位结构(图 2), 这种螺 旋状配位结构在开链冠醚的金属配合物 
表 3 非氢原子坐标和热参数 $\left(\times 10^{3} \mathrm{~nm}^{2}\right)$

\begin{tabular}{|c|c|c|c|c|}
\hline 原子 & $x$ & $y$ & $z$ & $B(\mathrm{eq})$ \\
\hline $\mathrm{Sr}$ & $0.73989(6)$ & $0.20778(6)$ & $0.73731(5)$ & $3.46(4)$ \\
\hline $\mathrm{O}(1)$ & $0.7636(4)$ & $0.2128(5)$ & $0.5990(4)$ & $4.9(4)$ \\
\hline $\mathrm{O}(2)$ & $0.6427(4)$ & $0.1234(4)$ & $0.6324(3)$ & $4.2(3)$ \\
\hline $\mathrm{O}(3)$ & $0.6724(4)$ & $0.0618(4)$ & $0.7738(4)$ & $4.7(4)$ \\
\hline $\mathrm{O}(4)$ & $0.7759(4)$ & $0.1470(4)$ & $0.8744(3)$ & $5.1(4)$ \\
\hline $\mathrm{O}(5)$ & $0.7818(4)$ & $0.3103(4)$ & $0.8475(4)$ & $4.8(4)$ \\
\hline $\mathrm{O}(6)$ & $0.8551(4)$ & $0.1150(4)$ & $0.7270(4)$ & $5.0(4)$ \\
\hline $\mathrm{O}(7)$ & $0.8717(5)$ & $0.2870(5)$ & $0.7142(4)$ & $5.9(4)$ \\
\hline $\mathrm{O}(8)$ & $0.6140(4)$ & $0.2551(5)$ & $0.7849(4)$ & $5.3(4)$ \\
\hline $\mathrm{O}(9)$ & $0.6882(4)$ & $0.3645(5)$ & $0.6991(4)$ & $5.4(4)$ \\
\hline $\mathrm{O}(10)$ & $0.9575(5)$ & $0.3364(5)$ & $0.6461(5)$ & $7.3(5)$ \\
\hline $\mathrm{O}(11)$ & $0.6622(5)$ & $0.4987(6)$ & $0.7074(5)$ & $8.2(6)$ \\
\hline $\mathrm{O}(12)$ & $1.1215(5)$ & $0.1656(7)$ & $0.5120(6)$ & $8.9(7)$ \\
\hline $\mathrm{O}(13)$ & $1.1200(5)$ & $0.0274(7)$ & $0.5220(5)$ & $7.8(6)$ \\
\hline $\mathrm{O}(14)$ & $0.9408(7)$ & $-0.1032(7)$ & $0.6941(7)$ & $10.7(8)$ \\
\hline $\mathrm{O}(15)$ & $0.8256(7)$ & $-0.0471(7)$ & $0.6704(7)$ & 11.1(8) \\
\hline $\mathrm{O}(16)$ & $0.4983(8)$ & $0.6195(7)$ & $0.8784(8)$ & $13.0(9)$ \\
\hline $\mathrm{O}(17)$ & $0.4283(6)$ & $0.5370(7)$ & $0.9441(6)$ & $9.0(7)$ \\
\hline $\mathrm{O}(18)$ & $0.481(1)$ & $0.2320(8)$ & $0.9486(7)$ & $16.0(1)$ \\
\hline $\mathrm{O}(19)$ & $0.4983(8)$ & $0.181(1)$ & $0.8604(9)$ & $15.0(1)$ \\
\hline $\mathrm{N}(1)$ & $0.7465(4)$ & $0.1848(5)$ & $0.4722(4)$ & $3.5(4)$ \\
\hline $\mathrm{N}(2)$ & $0.7994(5)$ & $0.3493(5)$ & $0.9704(4)$ & $4.4(5)$ \\
\hline $\mathrm{N}(3)$ & $0.9239(6)$ & $0.2744(7)$ & $0.6715(5)$ & $5.1(6)$ \\
\hline $\mathrm{N}(4)$ & $0.6538(5)$ & $0.4247(7)$ & $0.7270(5)$ & $5.0(5)$ \\
\hline $\mathrm{N}(5)$ & $1.0949(7)$ & $0.100(1)$ & $0.5357(6)$ & $6.9(8)$ \\
\hline $\mathrm{N}(6)$ & $0.8976(8)$ & $-0.0452(8)$ & $0.6751(7)$ & $7.1(8)$ \\
\hline $\mathrm{N}(7)$ & $0.4786(8)$ & $0.549(1)$ & $0.8986(8)$ & $8.7(9)$ \\
\hline $\mathrm{N}(8)$ & $0.526(1)$ & $0.238(1)$ & $0.9061(9)$ & $14.0(1)$ \\
\hline $\mathrm{C}(1)$ & $0.7270(6)$ & $0.1780(6)$ & $0.5457(6)$ & $4.0(5)$ \\
\hline$C(2)$ & $0.6569(7)$ & $0.1208(7)$ & $0.5551(5)$ & $5.2(6)$ \\
\hline $\mathrm{C}(3)$ & $0.6053(6)$ & $0.0450(7)$ & $0.6547(6)$ & $5.1(6)$ \\
\hline $\mathrm{C}(4)$ & $0.5966(7)$ & $0.0481(7)$ & $0.7354(7)$ & $5.9(7)$ \\
\hline $\mathrm{C}(5)$ & $0.6761(8)$ & $0.0422(7)$ & $0.8523(6)$ & $5.8(7)$ \\
\hline$C(6)$ & $0.7566(8)$ & $0.0585(7)$ & $0.8846(6)$ & $5.8(7)$ \\
\hline $\mathrm{C}(7)$ & $0.7732(6)$ & $0.1987(7)$ & $0.9394(5)$ & $4.6(5)$ \\
\hline $\mathrm{C}(8)$ & $0.7855(5)$ & $0.2891(7)$ & $0.9154(6)$ & $4.0(5)$ \\
\hline$C(11)$ & $0.8156(6)$ & $0.2352(6)$ & $0.4573(5)$ & $3.4(5)$ \\
\hline $\mathrm{C}(12)$ & $0.8277(6)$ & $0.3161(6)$ & $0.4867(6)$ & $4.3(6)$ \\
\hline$C(13)$ & $0.8967(8)$ & $0.3589(7)$ & $0.4708(7)$ & $5.5(7)$ \\
\hline$C(14)$ & $0.9487(7)$ & $0.325(1)$ & $0.4271(7)$ & $5.5(7)$ \\
\hline$C(15)$ & $0.9335(7)$ & $0.245(1)$ & $0.3969(7)$ & $6.0(7)$ \\
\hline$C(16)$ & $0.8675(6)$ & $0.1999(7)$ & $0.4116(6)$ & $5.0(6)$ \\
\hline$C(21)$ & $0.6989(6)$ & $0.1491(7)$ & $0.4081(5)$ & $4.1(6)$ \\
\hline $\mathrm{C}(22)$ & $0.6996(7)$ & $0.0626(8)$ & $0.3966(6)$ & $5.8(7)$ \\
\hline
\end{tabular}


续(表 3)

\begin{tabular}{lllll}
\hline 原子 & $x$ & \multicolumn{1}{c}{$y$} & \multicolumn{1}{c}{$z$} & $B(\mathrm{eq})$ \\
\hline $\mathrm{C}(23)$ & $0.6549(9)$ & $0.0278(9)$ & $0.3344(9)$ & $7.2(8)$ \\
$\mathrm{C}(24)$ & $0.6107(8)$ & $0.084(1)$ & $0.2871(7)$ & $7.0(1)$ \\
$\mathrm{C}(25)$ & $0.6126(7)$ & $0.169(1)$ & $0.2986(7)$ & $6.4(8)$ \\
$\mathrm{C}(26)$ & $0.6568(6)$ & $0.2043(8)$ & $0.3604(6)$ & $4.9(6)$ \\
$\mathrm{C}(31)$ & $0.8113(8)$ & $0.4381(7)$ & $0.9489(6)$ & $4.2(6)$ \\
$\mathrm{C}(32)$ & $0.7515(8)$ & $0.4854(8)$ & $0.9176(7)$ & $6.5(7)$ \\
$\mathrm{C}(33)$ & $0.765(1)$ & $0.572(1)$ & $0.8967(8)$ & $9.0(1)$ \\
$\mathrm{C}(34)$ & $0.840(1)$ & $0.603(1)$ & $0.909(1)$ & $9.0(1)$ \\
$\mathrm{C}(35)$ & $0.897(1)$ & $0.556(1)$ & $0.939(1)$ & $11.0(1)$ \\
$\mathrm{C}(36)$ & $0.883(1)$ & $0.471(1)$ & $0.9576(8)$ & $7.8(9)$ \\
$\mathrm{C}(41)$ & $0.7995(7)$ & $0.3286(6)$ & $1.0497(6)$ & $4.2(5)$ \\
$\mathrm{C}(42)$ & $0.8687(7)$ & $0.3006(8)$ & $1.0898(6)$ & $6.0(6)$ \\
$\mathrm{C}(43)$ & $0.8692(8)$ & $0.2820(8)$ & $1.1670(7)$ & $6.7(7)$ \\
$\mathrm{C}(44)$ & $0.800(1)$ & $0.296(1)$ & $1.2001(7)$ & $7.6(8)$ \\
$\mathrm{C}(45)$ & $0.7310(9)$ & $0.3242(8)$ & $1.1604(8)$ & $6.9(8)$ \\
$\mathrm{C}(46)$ & $0.7303(7)$ & $0.3387(7)$ & $1.0838(6)$ & $4.7(6)$ \\
$\mathrm{C}(51)$ & $0.9054(6)$ & $0.1138(7)$ & $0.6811(6)$ & $4.3(6)$ \\
$\mathrm{C}(52)$ & $0.9452(6)$ & $0.1886(7)$ & $0.6523(6)$ & $4.0(6)$ \\
$\mathrm{C}(53)$ & $1.0044(6)$ & $0.1824(8)$ & $0.6044(6)$ & $5.0(6)$ \\
$\mathrm{C}(54)$ & $1.0298(6)$ & $0.105(1)$ & $0.5835(6)$ & $5.0(7)$ \\
$\mathrm{C}(55)$ & $0.9961(7)$ & $0.0275(8)$ & $0.6072(6)$ & $5.6(7)$ \\
$\mathrm{C}(56)$ & $0.9351(7)$ & $0.0345(8)$ & $0.6528(7)$ & $5.2(6)$ \\
$\mathrm{C}(61)$ & $0.5845(6)$ & $0.3219(7)$ & $0.8081(6)$ & $4.2(6)$ \\
$\mathrm{C}(62)$ & $0.5991(6)$ & $0.4083(7)$ & $0.7838(6)$ & $3.9(5)$ \\
$\mathrm{C}(63)$ & $0.5640(7)$ & $0.4795(8)$ & $0.8124(6)$ & $5.4(6)$ \\
$\mathrm{C}(64)$ & $0.5115(7)$ & $0.4723(9)$ & $0.8657(7)$ & $5.5(7)$ \\
$\mathrm{C}(65)$ & $0.4969(6)$ & $0.391(1)$ & $0.8932(6)$ & $5.5(7)$ \\
$\mathrm{C}(66)$ & $0.5291(6)$ & $0.3203(7)$ & $0.8636(6)$ & $4.5(6)$ \\
\hline & & & &
\end{tabular}

表 4 合物 3 的部分键长 $(\mathrm{nm})$ 和键角 $\left({ }^{\circ}\right)$

\begin{tabular}{ll||cc}
\hline $\mathrm{Sr}-\mathrm{O}(6)$ & $2.455(7)$ & $\mathrm{O}(1)-\mathrm{Sr}-\mathrm{O}(2)$ & $59.1(2)$ \\
$\mathrm{Sr}-\mathrm{O}(8)$ & $2.485(7)$ & $\mathrm{O}(1)-\mathrm{Sr}-\mathrm{O}(6)$ & $75.4(2)$ \\
$\mathrm{Sr}-\mathrm{O}(1)$ & $2.533(7)$ & $\mathrm{O}(1)-\mathrm{Sr}-\mathrm{O}(7)$ & $67.7(2)$ \\
$\mathrm{Sr}-\mathrm{O}(5)$ & $2.579(6)$ & $\mathrm{O}(1)-\mathrm{Sr}-\mathrm{O}(9)$ & $78.5(2)$ \\
$\mathrm{Sr}-\mathrm{O}(7)$ & $2.623(8)$ & $\mathrm{O}(2)-\mathrm{Sr}-\mathrm{O}(3)$ & $60.3(2)$ \\
$\mathrm{Sr}-\mathrm{O}(4)$ & $2.638(6)$ & $\mathrm{O}(2)-\mathrm{Sr}-\mathrm{O}(6)$ & $96.0(2)$ \\
$\mathrm{Sr}-\mathrm{O}(3)$ & $2.652(7)$ & $\mathrm{O}(3)-\mathrm{Sr}-\mathrm{O}(4)$ & $62.4(2)$ \\
$\mathrm{Sr}-\mathrm{O}(9)$ & $2.660(8)$ & $\mathrm{O}(3)-\mathrm{Sr}-\mathrm{O}(6)$ & $83.1(2)$ \\
$\mathrm{Sr}-\mathrm{O}(2)$ & $\mathrm{O}(4)-\mathrm{Sr}-\mathrm{O}(5)$ & $59.5(2)$ \\
平均键长 & $2.718(6)$ & $\mathrm{O}(4)-\mathrm{Sr}-\mathrm{O}(6)$ & $74.8(2)$ \\
$\mathrm{Sr}-\mathrm{O}\left(\mathrm{C}-\mathrm{O}, \mathrm{Pic}{ }^{-}\right)$ & & $\mathrm{O}(5)-\mathrm{Sr}-\mathrm{O}(6)$ & $104.6(2)$ \\
$\mathrm{Sr}-\mathrm{O}(\mathrm{C}=\mathrm{O})$ & 2.470 & $\mathrm{O}(5)-\mathrm{Sr}-\mathrm{O}(7)$ & $69.3(2)$ \\
$\mathrm{Sr}-\mathrm{O}\left(\mathrm{NO}_{2}, \mathrm{Pic}{ }^{-}\right)$ & 2.556 & $\mathrm{O}(6)-\mathrm{Sr}-\mathrm{O}(7)$ & $64.2(2)$ \\
$\mathrm{Sr}-\mathrm{O}(\mathrm{C}-\mathrm{O}-\mathrm{C})$ & 2.642 & $\mathrm{O}(8)-\mathrm{Sr}-\mathrm{O}(9)$ & $62.6(2)$ \\
\hline
\end{tabular}


中尚属首例. 该结构与稀土配合物的 “类环状” 配位结构明显不同, 螯合链端基的伸展趋势不 是形成闭合环, 而是趋于分开, 5 个配位原子离 开垂直于螺旋轴并通过 $\mathrm{Sr}$ 原子的平面的距离 为: $\mathrm{O} 1,0.1123 ; \mathrm{O} 2,0.0010 ; \mathrm{O} 3,0.0000 ; \mathrm{O} 4$, -0.01234 ; O5, $-0.10414 \mathrm{~nm}$.

将该系列配合物与 TTD 的稀土苦味酸盐配 合物 ${ }^{[11,12]}$ 进行比较(表 5).

由表可以看出：(1) 在配体相同的条件下, 中心离子的配位数既与其离子半径有关又与其 电荷有关. 例如稀土离子 $\mathrm{La}(\mathrm{III}), \mathrm{Eu}(\mathrm{III}), \operatorname{Er}(\mathrm{III})$ 随着半径减小, 配位数减小; 而 $\operatorname{Sr}(\mathrm{II})$ 的半径大 于三价稀土离子, 但其配位数(9)却小于或等于 稀土离子的配位数(10 或 9). (2) 碱土金属配合 物与稀土配合物在晶系、空间群及配位多面体 等方面有较大的差异. (3) 稀土配合物均具有 “类环状” 配位结构, 而碱土金属配合物则为 螺旋状配位结构. 这种螺旋状鳌环具有较为固

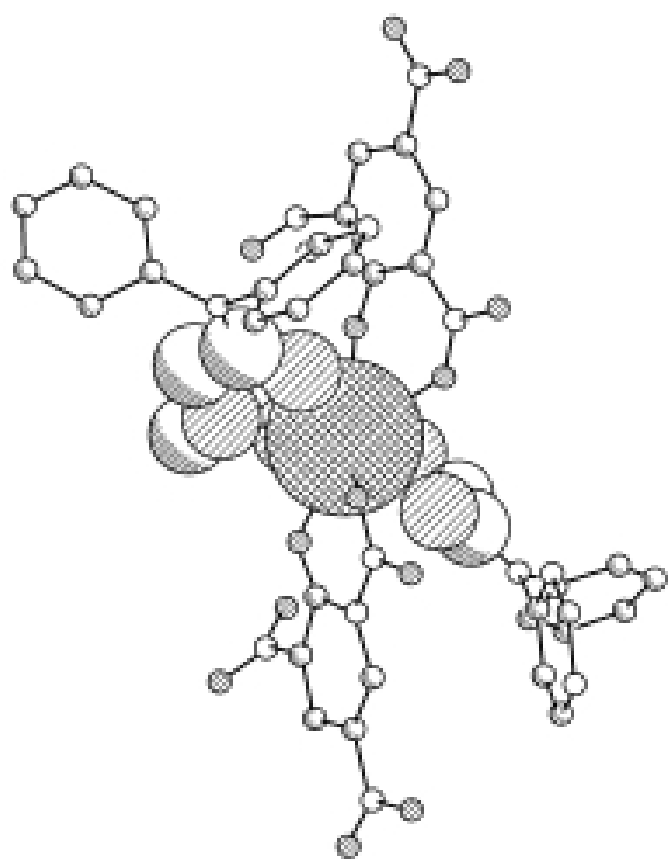

图 2 配合物 $\mathbf{3}$ 的螺旋状配位结构

表 5 TTD 的稀土及碱土金属配合物比较

\begin{tabular}{lcccc}
\hline & $\mathrm{Sr}(\mathrm{II})$ & $\mathrm{La}(\mathrm{III})$ & $\mathrm{Eu}(\mathrm{III})$ & $\operatorname{Er}(\mathrm{III})$ \\
\hline 离子半经 $/ \mathrm{nm}$ & 0.112 & 0.1061 & 0.0950 & 0.0881 \\
配位数 & 9 & 10,9 & 10,9 & 9 \\
晶系 & 单斜 & 正交 & 正交 & 三斜 \\
空间群 & $\mathrm{P} 2_{1} / \mathrm{a}$ & $\mathrm{Pcab}$ & $\mathrm{Pcab}$ & $\mathrm{Pi}$ \\
配位结构 & 螺旋状 & 类环状 & 类环状 & 类环状 \\
配位多面体 & 三帽三角棱柱 & $(10)$ 双帽四方棱柱 & $(10)$ 双帽四方棱柱 & 单帽四方反棱柱 \\
\hline
\end{tabular}

定的螺旋半径，对金属离子的选择性更高; 同时还具有手性(右手螺旋). 这或许是 TTD 对碱土 金属具有高选择性的原因.

\section{参考文献}

1 Güggi M, Pretsch E, Simon W. A barium ion-selective electrode based on the neutral carrier N, N, N', N'-tetraphenyl-3, 6, 9-trioxaundecane diamide. Anal Chim Acta, 1977, 91: 107 112

2 Ammann D, Bissig R, Güggi M, et al. Preparation of neutral ionophores for alkali and alkaline earth metal cations and their application in ion selective membrane electrodes. Helv Chim Acta, 1975, 58: 1535 1548

3 Ammann D, Pretsch E, Simon W. A synthetic, electrically neutral carrier for $\mathrm{Ca}^{2+}$. Tetrahedron Lett, 1972, 2473 2476

4 杨裕生, 丁玉珍, 谭干祖, 等. 链状聚醚用作萃取剂的研究. 核化学与放射化学, 1984, 6(4): 196 200

5 谭干祖, 徐浚哲, 焦天权. 多酰胺化合物的研究 II. 一种新的钡离子选择电极活性物质的合成, 有机化学, 1986, 2: 143 145 
6 Liu S X, Liu W S, Tan M Y, et al. Synthesis, characterization and structure of complexes of lanthanum (III) picrate with N, N, $\mathrm{N}^{\prime}, \mathrm{N}^{\prime}$-tetraphenyl-3, 6, 9-trioxaundecanediamide. J Coord Chem, 1996, 39: 105 115

7 Liu S X, Liu W S, Tan M Y, et al. Synthesis, and crystal structure of complexes of erbium (III) picrate with N, N, N', $\mathrm{N}^{\prime}$-tetraphenyl-3, 6, 9-trioxaundecanediamide. Polyhedron, 1997, 16(9): 1491 1495

8 Fan L Y, Liu W S, Liu S X, et al. Synthesis, crystal structure and fluroescence emission of the complexes of lanthanide picrates with N, N'-dimethyl-N,N'-diphenyl-3,6-dioxaoctanediamide. J Coord Chem, 1999, 48: 33 42

9 谭民裕, 柳士霞, 刘伟生, 等. 稀土苦味酸盐与 TDD 配合物的结构研究. 中国科学, B 辑, 1997, 27(2): 125 130

10 Geary W J. The use of conductivity measurements in organic solvents for the characterization of coordination compounds. Coord Chem Rev, 1971, 7: 81 122

11 覃诚真, 梁福沛, 何 晖, 等. 冠醚与轻稀土苦味酸盐配合物的合成和性质. 应用化学, 1991, 8 (4):77 79

12 毛希安, 沈联芳, 倪嘉缵. 稀土-苦味酸系列络合物 ${ }^{1}$ H NMR 研究. 波谱学杂志, 1985, 2: 105 109 